\title{
Dimensiones de Aprendizaje Para La Enseñanza De Riesgos Laborales en Enfermería
}

Andrés de Salomón Alas Díaz

Especialidad en Docencia Universitaria

Universidad Autónoma del Estado de México, Facultad de Enfermería y Obstetricia

andres.alasdiaz@gmail.com

Isabel Álvarez Solorza

Maestría en Ciencias de la Salud

Universidad Autónoma del Estado de México, Facultad de Enfermería y Obstetricia

isa_AlSo164@hotmail.com

Recibido:28-octubre-2019

Aceptado: 15 -enero-2020

\section{Introducción}

Los modelos educativos con los cuales el personal de enfermería se ha formado a lo largo del tiempo han pasado desde la instrucción del profesor puramente conductista, en donde el educando solamente seguía órdenes y memorizaba hasta lo que actualmente se conoce como constructivismo, donde el profesor funge como una guía para que el educando genere e integre el conocimiento de forma autorregulada. Actualmente existen diferentes modelos en la educación que resaltan esta perspectiva y conceptualizan al educando como sujeto constructor del conocimiento, capaz de tomar decisiones a través del análisis, la reflexión, integración y aplicación del conocimiento autorregulado. (Pecina,2013, p.1)

En la educación por competencias uno de los retos del profesor es posibilitar el aprendizaje significativo a través del acercamiento con la realidad. En la formación del profesional de enfermería el acercamiento a la realidad es a través de las prácticas clínicas, donde un actor clave en su aprendizaje son las (os) enfermeras (os) operativas (os) quien los orienta, pero que muchos de estos carecen de formación docente, lo que se considera una limitación para que el aprendizaje realmente sea significativo. (Pecina,2013, p.1)

Por lo que es fundamental que el profesional de enfermería clínico aprenda a enseñar, un modelo que es completo y que facilitaría el proceso de enseñanza en la práctica es el modelo de Robert Marzano. El fundamento de este modelo radica en que 
existen 5 dimensiones del aprendizaje, que son esenciales para un aprendizaje exitoso y permiten mantener el foco de atención del aprendizaje, estudiar este proceso y planear la evaluación asertiva tomando en cuenta las 3 esferas del aprendizaje (conocer, hacer y ser) y los 5 aspectos críticos de este. Cabe mencionar que, para poder indicar que el alumno ha alcanzado el aprendizaje de forma completa, indemne y autorregulado se debe alcanzar el desarrollo de la 5ta dimensión del aprendizaje, es decir, la conciencia del aprendizaje. (Marzano, 2005, p. 1-298)

En el siguiente trabajo tiene por objetivo determinar cómo el modelo de dimensiones de aprendizaje favorece la enseñanza de riesgos laborales en enfermería en un centro hospitalario de gineco pediatría; un problema presente en todas las instituciones hospitalarias. La OMS (2007), reportó que cerca de dos millones de trabajadores del sector salud a nivel mundial, han experimentado exposiciones percutáneas a enfermedades infecciosas anualmente. Las lesiones percutáneas constituyen la causa más común de exposición ocupacional a sangre y la principal causa de transmisión de agentes patógenos de la sangre, como es el Virus de Inmunodeficiencia Humana (VIH), Hepatitis, entre otros.

El Consejo internacional de enfermeras (ICN) acepta que todas las enfermeras tienen derecho a trabajar en un entorno saludable y seguro, sin riesgo de lesiones o enfermedades derivadas de su trabajo. Así mismo promueve el desarrollo y la aplicación de políticas o instrumentos internacionales, nacionales y locales que protejan el derecho de las enfermeras a un entorno de trabajo seguro, incluyendo formación continua que integre nuevos modelos de enseñanza aprendizaje y permitan la investigación en el área de la salud y la seguridad laboral (Consejo Internacional de Enfermeras, 2017).

\section{El aprendizaje}

El aprendizaje ha sido definido a lo largo del tiempo por diferentes autores, sin tener actualmente una definición aceptada internacionalmente, sin embargo, Shuell define al aprendizaje como: "un cambio perdurable en la conducta o en la capacidad de comportarse de una determinada manera, la cual resulta de la práctica o de alguna otra forma de experiencia". Dicha definición nos acerca a la forma en que se da el aprendizaje en enfermería, el cual como se sabe es una mezcla entre el conocimiento, la práctica y la experiencia. 


\section{El aprendizaje conductista y constructivista}

El aprendizaje, así como la sociedad, ha ido evolucionando en función de la época y las situaciones que aquejan a las diferentes sociedades alrededor del mundo, explicándose y expresándose a través de diferentes teorías del aprendizaje, pasando desde la etapa memorística (conductismo) hasta la etapa del aprendizaje autorregulado e independiente (constructivismo).(Peggy, 1993, p.52-73)

El aprendizaje conductista fundado por Watson en 1913 está centrado en el principio de que para aprender deben existir estímulos previos y que la respuesta debe ser la misma, es decir que para aprender debe utilizarse la memorización repetitiva y mecánica. Este tipo de aprendizaje por parte del docente centra su instrucción en que el docente es el máximo exponente del conocimiento y dueño de "la verdad absoluta", sin permitir la innovación o creación del conocimiento y centrándose en objetivos previamente establecidos, que no permite el enriquecimiento por parte de los alumnos, y dedicándose exclusivamente a la memorización del contenido sin analizar reflexionar y procesar la información. Un ejemplo claro de esto es la forma en que en la década de los 90’s aprendíamos las tablas de multiplicar, repitiendo la misma información sin parar a través inclusive de canciones (estímulos) y obteniendo la misma respuesta una y otra vez. (Peggy, 1993, p.52-73)

\section{El aprendizaje constructivista}

El aprendizaje constructivista tienes sus orígenes con Kant y Vico y centra sus instrucciones en que el conocimiento es una construcción del ser humano: cada persona percibe su realidad, la organiza y la interpreta en forma de constructos. En este tipo de aprendizaje, el ser humano necesita de todas su s habilidades y destrezas para organizar e integrar una tarea. Para Piaget llamado asimilación y acomodación. En el aprendizaje constructivista este se da a través de 3 procesos: lógicos, cognitivos y afectivos haciendo asimilación con los 3 saberes de la educación: el conocer, el hacer y el ser. Para este aprendizaje, el estudiante es el centro del proceso y el profesor funge solamente como una guía para el proceso de enseñanza aprendizaje con la libertad de modificar en el momento que considere pertinente y de acuerdo con el avance del alumno, a crear nuevos escenarios de educación y nuevas estrategias de aprendizaje, en donde el estudiante se 
dedica a autorregularse y aprender de forma autónoma, desarrollando las tres esferas del aprendizaje y obteniendo conciencia de este. (Ortiz, 2015. P.93-110)

\section{Modelo de Dimensiones de Aprendizaje de Robert Marzano}

El modelo de dimensiones de aprendizaje de Robet Marzano, es un modelo muy completo que hace uso de lo que los profesores saben ya acerca del aprendizaje para definir su proceso de enseñanza. El fundamento de este modelo radica en que existen 5 dimensiones del aprendizaje, que son esenciales para un aprendizaje exitoso y permiten: Mantener el foco de atención del aprendizaje, estudiar este proceso y planear la evaluación asertiva tomando en cuenta las 3 esferas del aprendizaje (conocer, hacer y ser) y los 5 aspectos críticos de este. Cabe mencionar que, para poder indicar que el alumno a alcanzado el aprendizaje de forma completa, indemnemente y auto regulada se debe alcanzar el desarrollo de la 5ta dimensión del aprendizaje, es decir, la conciencia del aprendizaje.

\section{Dimensión 1: Actitudes y percepciones}

En esta dimensión se busca que el alumno se motive en relación con el tema a abordar. Las aptitudes y percepciones afectan de forma directa el aprendizaje del alumno, si este se siente inseguro, es probable que no aprenda de forma eficaz. Por ello un elemento clave para el aprendizaje del alumno, es la motivación en todos los escenarios donde este se desarrolla.

Para desarrollar la dimensión 1: Actitudes y percepciones, además de las actividades mencionadas con anterioridad, existen una gran variedad de estrategias de enseñanzaaprendizaje que ayudan para el desarrollo de esta dimensión, como lo son: las actividades focales introductorias, por ejemplo: Anécdotas, juegos, noticias, fragmentos de lectura, experiencias. También se pueden utilizar preguntas guía literales o exploratorias, Lluvia de ideas, discusiones guiadas, entre otros, es decir actividades, que, de un inicio, dispongan e integren positivamente a los alumnos para el aprendizaje.

\section{Dimensión 2: Adquirir e integrar el conocimiento}

Esta dimensión busca que el alumno adquiera nuevos conocimientos a partir de lo que ya sabe. Cuando los alumnos están adquiriendo información nueva se les debe dirigir para que la relacionen con sus conocimientos previos; es decir el aprendizaje significativo. 
Para que el aprendizaje sea significativo con relación a conocimientos, habilidades destrezas y actitudes, se le debe dirigir al alumno a través de un modelo o una serie de pasos para que el conocimiento, la habilidad o actitud sea eficiente, se pueda interiorizar y por último desempeñarse con facilidad.

Las estrategias de enseñanza aprendizaje para el desarrollo de la dimensión 2 pueden ser: Discursos, Lecturas, Exposiciones con diferentes tipos de señalizaciones, Ilustraciones, gráficos, preguntas previas, organizadores gráficos como el mapa conceptual y mental, líneas del tiempo, SQA, entre otros; es decir estrategias que permitan adquirir, conocimiento de tipo declarativo y procedimental al alumno.

\section{Dimensión 3: Extender y refinar el conocimiento}

El aprendizaje no se detiene con la adquisición del conocimiento (Dimensión 2), sino que este debe ser procesado, es decir, extendido y refinado como Marzano lo menciona. Esta dimensión busca, una vez que se ha adquirido el conocimiento, que este sea analizado de forma rigurosa a través de proceso de razonamiento, para entender y refinar la información. Las estrategias educativas para desarrollar esta dimensión pueden ser actividades de representación del lenguaje como: Organizadores gráficos como el cuadro comparativo y la matriz de clasificación y tareas estructuradas como el resumen y el listado de ideas principales.

\section{Dimensión 4: Uso significativo del conocimiento}

Para demostrar que el aprendizaje es efectivo, se debe identificar el uso del conocimiento en el desarrollo de tareas específicas. Asegurarse de que el alumno utilice el conocimiento de forma significativa en un contexto que tenga sentido para ellos, es la finalidad de la dimensión 3. Cuando los alumnos usan el conocimiento en contextos auténticos, el nivel de compromiso aumenta. Muchos alumnos solamente cumplen con sus tareas por que se les ha asignado, sin embargo, en el momento que se le encuentra sentido y utilidad al conocimiento, se logra un nivel más alto de comprensión y desarrollo de las habilidades.

Las estrategias educativas para el desarrollo de la dimensión 4 son: la simulación, el estudio de caso, aprendizaje basado en problemas, método de proyectos, aprendizaje in 
situ, aprendizaje basado en tecnologías de la información, investigación con tutoría, entre otros.

\section{Dimensión 5: Hábitos mentales}

La dimensión 5 tiene como finalidad crear en el alumno hábitos mentales que le permitan crear conciencia del aprendizaje, para ello, debe pensar de forma crítica, creativa y autorregulada y así, controlar su comportamiento.

Las estrategias de aprendizaje para desarrollar esta dimensión pueden ser, estrategias metacognitivas de supervisión y evaluación como: Debate, Hoja del recuerdo, historia de clase, recuerdos, línea de aprendizaje, termómetro de avance y preguntas de metacognición, entre otros.

En conclusión, si se logra el desarrollo de todas las dimensiones de aprendizaje hasta la conciencia de este (dimensión 5), se habrá cumplido el objetivo del modelo de dimensiones: la optimización del aprendizaje de los alumnos de forma crítica, creativa y autorregulada. (Marzano, 2015 p.1-298)

\section{Objetivo general}

Determinar cómo las dimensiones de aprendizaje favorecen la enseñanza de riesgos laborales en enfermería.

\section{Objetivos específicos}

- Diseñar un instrumento para medir las dimensiones de aprendizaje en el profesional de enfermería.

- Diseñar un instrumento para medir el nivel de conocimientos en riesgos laborales en el profesional de enfermería.

- Evaluar la validez de constructo y confiabilidad de los instrumentos elaborados.

- Identificar las formas de enseñanza del profesional de enfermería a través del modelo de dimensiones de aprendizaje.

- Evaluar el nivel de conocimientos que tiene el profesional de enfermería sobre riesgos laborales. 
- Establecer intervenciones para favorecer el desarrollo de la enseñanza a través del modelo de dimensiones de aprendizaje en el profesional de enfermería.

- Establecer intervenciones para que el profesional de enfermería eleve el nivel de conocimientos en riesgos laborales a través de las dimensiones de aprendizaje.

- Evaluar las intervenciones realizadas en el profesional de enfermería para el desarrollo de las dimensiones de aprendizaje y favorecer el nivel de conocimientos enseñanza de riesgos laborales.

\section{Metodología}

\section{Universo de estudio}

Profesional de enfermería del Hospital de Gineco Pediatría 3A del Instituto Mexicano del seguro social (IMSS). Para el profesional de enfermería se realiza un muestreo no probabilístico a conveniencia con una población total de 203 enfermeras del turno matutino y vespertino de las diferentes categorías, de las cuales se obtiene una muestra total de $\mathrm{N}=40$. A partir de esta submuestra se establecerá un grupo control y de intervención.

\section{Instrumentos}

Para la medición de dimensiones de aprendizaje, se diseñó un instrumento a través de las estrategias y formas de enseñanza que utiliza el profesional de enfermería para la docencia en práctica clínica, basándonos en el modelo de dimensiones de aprendizaje de Robert Marzano, el cual consta de 45 ítems con escala de respuesta tipo Likert. Dicho instrumento se validó a través de expertos nacionales e internacionales con un pilotaje de 30 cuestionarios. (técnica Delphi). Alpha de Cronbach 0.94

Además, se diseñó un cuestionario para evaluar el nivel de conocimientos sobre riesgos laborales de 26 ítems con opción de respuesta tipo multirreactivo, el cual consta de 26 items, basado en criterios nacionales, internacionales e institucionales, validado por expertos nacionales (técnica Delphi). Alpha de Cronbach 0.84

\section{Recolección de datos}


Para la recolección de datos, posterior a la aceptación por el comité de ética de la institución hospitalaria donde se aplicará el estudio, se le invitó al profesional de enfermería a participar en el estudio a través de la firma de consentimiento informado.

El levantamiento de los datos se realizará en tres fases:

\section{Primer Etapa:}

Diagnóstico. Profesional de Enfermería. Se aplicó un cuestionario para identificar las dimensiones de aprendizaje, así como un cuestionario sobre conocimientos de riesgos laborales.

\section{Intervención.}

1. Implementación de un taller para desarrollar las dimensiones de aprendizaje a partir del modelo de dimensiones de aprendizaje de Robert Manzano.

2. Taller sobre riesgos laborales a partir del modelo de dimensiones de aprendizaje de Robert Manzano.

\section{Evaluación.}

Se aplicará nuevamente el cuestionario para identificar las dimensiones de aprendizaje, así como el cuestionario sobre conocimientos de riesgos laborales.

\section{Análisis de datos}

Se realizará una descripción univariada para las variables categóricas con proporciones, las variables cuantitativas se reportarán con promedio y desviación estándar. En el análisis bivariado, se evaluará la asociación cruda entre las variables independientes y la variable dependiente, mediante la prueba chi2 para variables categóricas y la t-student para variables continuas. Además, se hará un análisis de sensibilidad que evalúe la capacidad del cuestionario para detectar un cambio en el nivel de conocimientos en las poblaciones estudio. Para ello, se utilizará el software Stata 15.

\section{Aspecto ético}

Para poder participar en el estudio, se firmará el consentimiento informado, de acuerdo con el artículo 100 de la ley general de salud de los estados unidos mexicanos.

\section{Resultados}


Los siguientes resultados corresponden a la primera fase del estudio; es decir la etapa de diagnostico y de diseño de instrumentos de evaluación.

\begin{tabular}{llll}
\hline Tabla 1. Datos Sociodemográficos & & \\
\hline Edad & 23 a 62 & Media & 37 años \\
Antigüedad & 6 m a 30 años & Media & 11 años \\
& & & \\
Sexo & $10 \%$ Hombres & $90 \%$ Mujeres & \\
Servicio & Ginecología & $37 \%$ & \\
& & & \\
Tipo de contratación & Base & $85 \%$ & \\
Grado de estudios & Técnico 50\% & Licenciatura & $37.50 \%$ \\
Cursos de formación & 32.5 Sí & Monográfico & $17.50 \%$ \\
\hline
\end{tabular}

En la siguiente tabla podemos observar que el rango de edad del profesional de enfermería fue de 23 hasta los 62 años, con una media de 37 años. La antigüedad laboral obtuvo un rango desde los 6 meses hasta los 30 años de servicio. El sexo de predominio fueron las mujeres con un $90 \%$. El servicio que predomino en participación fue el de ginecología con un 37\%. El tipo de contratación fue el de "base" con un 85\% y un 15\% de contrato 02 y 08 . El grado de estudios fue mayormente técnico en enfermería con un $50 \%$ mientras que la licenciatura solo un37.5\%, cabe mencionar que los estudios de posgrado como especialidad maestría y doctorado se encontraron nulos $(0 \%)$.

Finalmente podemos observar que el profesional de enfermería, en su mayoría (62.5\%) no cuenta con cursos de formación docente de distinta índole, reportando solo asi un $32.5 \%$ que si a tomado algún curso de formación docente en su mayoría de tipo monográfico. 
Tabla 2. Dimensiones de Aprendizaje de Robert Marzano

\begin{tabular}{|c|c|c|c|c|c|}
\hline \multirow[b]{2}{*}{ Ítem } & \multicolumn{5}{|c|}{ Respuestas } \\
\hline & 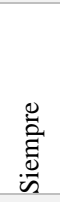 & 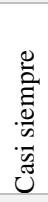 & 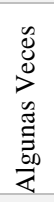 & $\begin{array}{l}N \\
\stackrel{N}{\pi} \\
\approx \\
\approx\end{array}$ & $\begin{array}{c}\tilde{U} \\
\Xi \\
Z\end{array}$ \\
\hline \multicolumn{6}{|l|}{ Dimensión 1 Actitudes y percepciones } \\
\hline $\begin{array}{l}\text { 1. ¿Para motivar el aprendizaje del alumno, utilizas actividades como anécdotas, juegos, noticias, } \\
\text { fragmentos de lectura, experiencias y/o videos? }\end{array}$ & 8 & 13 & 12 & 4 & 3 \\
\hline $\begin{array}{l}\text { 2. ¿Utilizas preguntas guía, literales o exploratorias, o discusiones guiadas, para motivar el aprendizaje } \\
\text { del alumno? }\end{array}$ & 5 & 22 & 9 & 3 & 1 \\
\hline 3. ¿Consideras que mantienes una actitud positiva hacia el alumno? & 20 & 18 & 2 & 0 & 0 \\
\hline $\begin{array}{l}\text { 4. ¿Compartes experiencias con el alumno y/o historias de personajes famosos o conocidos donde } \\
\text { destacas cómo una buena actitud influye directamente en su formación profesional? }\end{array}$ & 7 & 19 & 9 & 4 & 1 \\
\hline 5. ¿Conversas y/o saludas al alumno, antes, durante y después de la práctica? & 29 & 10 & 1 & 0 & 0 \\
\hline 6. ¿Identificas a los alumnos que tienen problemas para aprender? & 13 & 19 & 6 & 1 & 1 \\
\hline 7. ¿Consideras que tratas a los alumnos de forma imparcial y positiva? & 25 & 14 & 1 & 0 & 0 \\
\hline $\begin{array}{l}\text { 8. ¿Identificas las formas de aprender de cada alumno y elaboras estrategias de aprendizaje acordes con } \\
\text { sus formas de aprender? }\end{array}$ & 4 & 14 & 10 & 12 & 0 \\
\hline 9. ¿Consideras que tienes apertura para que los alumnos utilicen sus propias estrategias de aprendizaje? & 10 & 19 & 6 & 5 & 0 \\
\hline $\begin{array}{l}\text { 10. ¿Estableces y comunicas las reglas, normas y políticas de seguridad para el aprendizaje en el } \\
\text { servicio durante la práctica clínica? }\end{array}$ & 18 & 16 & 5 & 1 & 0 \\
\hline 11. ¿Permites agresiones verbales entre los alumnos durante la práctica clínica? & 0 & 1 & 0 & 1 & 38 \\
\hline \multicolumn{6}{|l|}{ Dimensión 2. Adquirir e Integrar el conocimiento } \\
\hline 12. ¿Utilizas la exposición oral para que el alumno adquiera conocimiento? & 5 & 22 & 9 & 3 & 1 \\
\hline 13. ¿Ayudas al alumno a comprender el sentido e importancia del conocimiento teórico? & 16 & 20 & 4 & 0 & 0 \\
\hline 14. ¿Solicitas al alumno que realice búsqueda de información y ofreces opciones para ello? & 17 & 18 & 4 & 1 & 0 \\
\hline 15. ¿Proporcionas al alumno preguntas guía para que realice organizadores gráficos? & 8 & 12 & 9 & 8 & 3 \\
\hline 16 ¿Solicitas al alumno representaciones gráficas, pictográficas y tablas en sus tareas y/o exposiciones? & 1 & 12 & 11 & 10 & 6 \\
\hline 17. ¿Utilizas nemotecnias para la enseñanza? & 4 & 8 & 13 & 6 & 9 \\
\hline 18. ¿Cuándo enseñas al alumno una habilidad o procedimiento, hablas en voz alta? & 18 & 11 & 7 & 4 & 0 \\
\hline 19. ¿Consideras que identificas con el alumno las habilidades qué desarrolla? & 12 & 16 & 9 & 2 & 1 \\
\hline $\begin{array}{l}\text { 20. ¿Ayudas al alumno a realizar un repaso mental de los pasos para desarrollar un procedimiento y } \\
\text { posteriormente demuestras cómo realizarlo? }\end{array}$ & 11 & 19 & 6 & 3 & 1 \\
\hline $\begin{array}{l}\text { 21. ¿Creas oportunidades para que el alumno practique habilidades y procedimientos y le señalas sus } \\
\text { errores al realizarlos? }\end{array}$ & 13 & 19 & 5 & 2 & 1 \\
\hline \multicolumn{6}{|l|}{ Dimensión 3. Extender y Refinar el Conocimiento } \\
\hline 22. ¿Utilizas el mapa comparativo y/o la matriz de clasificación para la enseñanza? & 2 & 10 & 11 & 7 & 10 \\
\hline 23. ¿Cuándo enseñas un tema lo realizas de forma inductiva y/o deductiva? & 4 & 19 & 9 & 5 & 3 \\
\hline 24. ¿Identificas deficiencias de conocimiento en el alumno al realizar procedimientos? & 12 & 17 & 9 & 2 & 0 \\
\hline 25. ¿Solicitas al alumno la elaboración de resúmenes y/o un listado de ideas principales? & 9 & 10 & 10 & 7 & 4 \\
\hline 26. ¿Identificas con el alumno diferentes perfectivas de un tema y examinan lógicamente cada una? & 3 & 18 & 10 & 5 & 4 \\
\hline
\end{tabular}


Tabla 2. Dimensiones de Aprendizaje de Robert Marzano

\begin{tabular}{|c|c|c|c|c|c|}
\hline \multirow[b]{2}{*}{ Ítem } & \multicolumn{5}{|c|}{ Respuestas } \\
\hline & 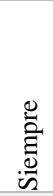 & 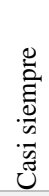 & 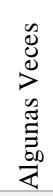 & 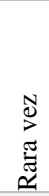 & $\stackrel{\widetilde{J}}{\Xi}$ \\
\hline \multicolumn{6}{|l|}{ Dimensión 4. Uso Significativo Del Conocimiento } \\
\hline $\begin{array}{l}\text { 27. ¿Desarrollas estrategias o productos únicos que satisfagan la deficiencia de aprendizaje detectada en el } \\
\text { alumno? }\end{array}$ & 7 & 12 & 10 & 6 & 5 \\
\hline $\begin{array}{l}\text { 28. ¿Utilizas la simulación, los casos clínicos y/o el aprendizaje basado en problemas para enseñarle al } \\
\text { alumno? }\end{array}$ & 10 & 13 & 11 & 5 & 1 \\
\hline 29. ¿Utilizas el aprendizaje in situ para la enseñanza con él alumno? & 5 & 16 & 8 & 7 & 4 \\
\hline 30. ¿Utilizas la investigación con tutoría para enseñarle al alumno? & 8 & 13 & 9 & 4 & 6 \\
\hline $\begin{array}{l}\text { 31. ¿Utilizas el análisis con el alumno para conocer cómo interactúan entre si los conocimientos, habilidades } \\
\text { y procedimientos aprendidos? }\end{array}$ & 4 & 21 & 8 & 5 & 2 \\
\hline \multicolumn{6}{|l|}{ Dimensión 5. Hábitos Mentales } \\
\hline 32. ¿Supervisas al alumno durante su aprendizaje? & 24 & 12 & 3 & 0 & 1 \\
\hline 33. ¿Consideras que tus alumnos buscan la precisión en el desarrollo de procedimientos? & 8 & 18 & 11 & 2 & 1 \\
\hline 34. ¿Consideras que tus alumnos actúan de forma impulsiva en el desarrollo de procedimientos? & 1 & 5 & 26 & 4 & 4 \\
\hline 35. ¿Consideras que tu alumno responde y actúa de acuerdo al nivel de estudios en el que se está preparando? & 5 & 17 & 13 & 4 & 1 \\
\hline 36. ¿Consideras que tu alumno reflexiona sobre su aprendizaje? & 6 & 19 & 10 & 3 & 2 \\
\hline 37. ¿Consideras que tu alumno responde de con una actitud positiva cuando le brindas retroalimentación? & 12 & 19 & 6 & 2 & 1 \\
\hline 38. ¿Facilitas la discusión positiva durante la práctica clínica? & 11 & 16 & 10 & 2 & 1 \\
\hline 39. ¿Solicitas a los alumnos que identifiquen y manifiesten las habilidades que les gustaría desarrollar? & 10 & 15 & 10 & 3 & 2 \\
\hline 40. ¿Consideras que eres un modelo a seguir para tus alumnos? & 9 & 12 & 14 & 3 & 2 \\
\hline 41. ¿Identificas en el alumno sus habilidades y le solicitas que se concentre en perfeccionarlas? & 10 & 21 & 7 & 1 & 1 \\
\hline 42. ¿Solicitas entre los alumnos que se supervisen para el desarrollo de sus habilidades? & 10 & 16 & 9 & 1 & 4 \\
\hline 43. ¿Solicitas al alumno que autoevalúe su aprendizaje? & 8 & 12 & 13 & 4 & 3 \\
\hline 44. ¿Brindas al alumno retroalimentación de su desempeño a través de anecdotarios o reportes? & 11 & 12 & 12 & 5 & 3 \\
\hline 45 ¿Utilizas preguntas de meta cognición para evaluar el desarrollo de hábitos mentales en el alumno? & 6 & 15 & 7 & 3 & 9 \\
\hline
\end{tabular}

En relación con el desarrollo de dimensiones de aprendizaje por el profesional de enfermería, podemos observar que ninguna dimensión se desarrolla de forma completa (dimensiones 1 a 5). El mayor número de respuestas de las dimensiones 1 a 4 están concentradas en "casi siempre" mientras que para la dimensión 5 Hábitos mentales se concentran en "algunas veces".

\section{Discusión}


El modelo de dimensiones de aprendizaje de Robot Marzano es un modelo muy completo que hace uso de lo que los profesores saben ya acerca del aprendizaje para definir su proceso de enseñanza. El fundamento de este modelo radica en que existen 5 dimensiones del aprendizaje, que son esenciales para un aprendizaje exitoso y permiten: Mantener el foco de atención del aprendizaje, estudiar este proceso y planear la evaluación asertiva tomando en cuenta las 3 esferas del aprendizaje (conocer, hacer y ser) y los 5 aspectos críticos de este modelo. Dicho modelo inicio desde los 90s, más sin embargo el $97.5 \%$ del personal de enfermería no lo conoce mientras que solo un $2.5 \%$ sí.

En este estudio podemos visualizar que de la dimensión 1 a 3 el profesional de enfermería utiliza "casi siempre" estrategias de aprendizaje para el desarrollo estas dimensiones. Dicho de otra forma, podemos identificar que el profesional de enfermería "casi siempre" hablando de la dimensión 1 motiva al alumno al aprendizaje a través de anécdotas, historias, juegos, conversaciones y trata a los alumnos de forma imparcial. Así mismo con relación a la dimensión 2 (adquisición e integración del conocimiento) “casi siempre el profesional de enfermería utiliza para la enseñanza: la exposición oral, ayuda a 1 alumno a entender la importancia teórica del conocimiento, proporciona preguntas guía para la elaboración de organizadores gráficos, utiliza nemotecnias para la enseñanza, identifica en el estudiante las habilidades que desarrolla y crea oportunidades para que el alumno practique habilidades; mientras que por otro lado, siempre habla en voz alta para la enseñanza.

Por otro lado, en lo que respecta a la dimensión 3. Refinar y extender el conocimiento. Marzano menciona que para el cumplimiento de esta dimensión se necesitan desarrollar procesos de pensamiento como: comparar, clasificar, razonar de forma inductiva y deductiva y analizar errores y perspectivas, lo cual en este estudio identificamos que por parte del profesional de enfermería "casi siempre" y "algunas veces" se desarrollan.

En la dimensión 4. Uso significativo del conocimiento, Se identifican 6 procesos de razonamiento para darle sentido al uso del conocimiento, diferentes a los que se utilizan solo para recordar información (dimensión 2), estos son: La toma de decisiones, solución de problemas, la invención, indagación experimental, investigación y análisis de sistemas, los cuales se pueden conseguir a través de estrategias como: la simulación, el 
aprendizaje basado en problemas, aprendizaje in situ, análisis de casos e investigación con tutoría. Dichas estrategias son utilizadas por el profesional de enfermería en su mayoría “casi siempre” mermando así el desarrollo completo de la cuarta dimensión.

Para que el alumno alcance el aprendizaje de forma completa y auto regulada se debe alcanzar el desarrollo de la 5ta dimensión del aprendizaje, es decir, la conciencia del aprendizaje o mejor conocida como hábitos mentales., lo cual podemos observar en este estudio no se alcanza y/o desarrolla completamente; ya que el mayor número de respuestas de los participantes en este caso el profesional de enfermería de las diferentes categorías antes mencionadas se concentran en la respuesta "algunas veces", observando así que el profesional de enfermería "algunas veces" utiliza estrategias de enseñanza para el desarrollo de hábitos mentales como: la supervisión, la búsqueda de la precisión en el desarrollo de procedimientos, la respuesta del estudiante de acuerdo al nivel de conocimientos, la identificación de habilidades para su desarrollo, la autoevaluación y las preguntas de metacognición, entre otras.

Por otro lado, Garduño en un estudio realizado en en 2012 menciona que el profesional de enfermería sabe del riesgo a los que se expone, pero un problema importante es la falta de conciencia o una mayor preocupación por su seguridad y el uso de equipo o estrategias para reducir los riesgos lo cual concuerda con este estudio ya que a pesar de que la mayoría de los profesionales de enfermería cuentan con un nivel de conocimientos ALTO, no conocen de forma clara los procedimientos a seguir en caso de sufrir un accidente laboral derivado de sus funciones. Así mismo tienen problemas para identificar y clasificar sus actividades diarias según el tipo de riesgo al que se exponen.

En un estudio de tipo cuantitativo, descriptivo, retrospectivo, realizado por Sergio, A. Et Al. Se presentó un análisis sobre accidentes de trabajo y de trayecto, del año 2009 en un hospital de seguridad social en el estado de Jalisco con el objetivo de evaluar accidentabilidad, dándose una accidentabilidad de 106 riesgos de trabajo de los cuales 66 fueron accidentes de trabajo y 49 accidentes de trayecto, en dicho periodo. En la categoría de enfermera general, los accidentados de trabajo como de trayecto, fue la de mayor accidentabilidad con un $25.50 \%$. lo anterior por ser la plantilla más grande de la institución lo cual en ese sentido coincide con este estudio al ser la enfermera general la 
categoría predominante, más, sin embargo, en su mayoría no conoce la diferencia entre una enfermedad profesional y un accidente de trabajo.

Finalmente, el Consejo internacional de enfermeras (ICN) se desempeña como la organización internacional que establece y fomenta el desarrollo y aplicación de políticas o instrumentos internacionales, nacionales y locales que protegen el derecho de las enfermeras a un entorno de trabajo seguro, incluyendo formación continua y anima a la investigación en el área de la salud y la seguridad laboral, por lo cual todo profesional de enfermería debería conocerlo, lo cual podemos identificar que no es así, ya que el profesional desconoce cuáles son los organismos nacionales e internacionales regulatorios de los riesgos laborales en enfermería alejándolo de información y políticas útiles para la prevención de riesgos laborales.

\section{Conclusiones}

El profesional de enfermería no utiliza "siempre" estrategias que permitan un aprendizaje exitoso, se centra "casi siempre" en estrategias para adquirir procesar y aplicar el conocimiento, pero resta importancia al desarrollo de hábitos mentales utilizando solo "algunas veces" estrategias para el desarrollo de estos. Por lo cual, se sugiere formación en docencia para así utilizar métodos y estrategias de enseñanza exitosas y lograr en el estudiante de práctica clínica el aprendizaje autorregulado.

\section{Referencias}

Peggy, A. Et Al. Conductismo, cognitivismo y constructivismo: una comparación de los aspectos críticos desde la perspectiva del diseño de instrucción. (internet). 1993. (citado 2018 Nov o1). Performance Improvement Quarterly, 1993, 6(4):50-72. Disponible en: http://www.galileo.edu/pdh/wpcontent/blogs.dir/4/files/2011/05/1.-ConductismoCognositivismo-y-

Constructivismo.pdf

Ortiz Granja, D. El constructivismo como teoría y método de enseñanza. Sophia, Colección de Filosofía de la Educación [Internet]. 2015;(19):93-110. Recuperado de: http://www.redalyc.org/articulo.oa?id=441846096005 
Marzano, R., 2005. Dimensiones Del Aprendizaje. Manual Del Maestro. Segunda Ed. Jalisco: Iteso. P.

Bernal, M. L. Propuesta para la enseñanza del cuidado en Enfermería. Enfermería Universitaria ENEO-UNAM. [Internet]. 2009[citado 27 Ago 2019]; 6(1), 33-41. Disponible en: https://www.medigraphic.com/pdfs/enfuni/eu-2009/eu091e.pdf

Elia A.C., Orteguin R.M. La enseñanza clínica en enfermería: un acercamiento multidisciplinario. IPN. México, DF; junio de 2007; Consultado el 7 de octubre de 2019 .

Vera Regina W. Enseñanza de enfermería centrada en el cuidado. Aquichan, [Internet]. 2010 [citado 26 Ago 2019]; 9(3): [aprox. 0 p.]. Disponible en: http://aquichan.unisabana.edu.co/index.php/aquichan/article/view/1523/1970

Sánchez Rodríguez JR. Preparación pedagógica, docentes clínicos de enfermería y su relación con el proceso-enseñanza aprendizaje práctico. Rev Cubana Enferm [Internet]. 2017 [citado 26 Ago 2019]; 33(4): [aprox. 0 p.]. Disponible en: http://www.revenfermeria.sld.cu/index.php/enf/article/view/2131

Moya J L, Parra S. La enseñanza de la enfermería como una práctica reflexiva. Texto contexto - enferm. [Internet]. 2006 [cited 2019 Aug 26]; 15(2): 303-311. Disponible en: http://www.scielo.br/scielo.php?script=sci_arttext\&pid=S010407072006000200015\&lng=en. http://dx.doi.org/10.1590/S010407072006000200015

Pérez C, Alameda A, Albéniz C. La formación práctica en enfermería en la Escuela Universitaria de Enfermería de la Comunidad de Madrid. Opinión de los alumnos y de los profesionales asistenciales: Un estudio cualitativo con grupos de discusión. Rev. Esp. Salud Pública [Internet]. 2002 [citado 2019 Ago 26]; 76(5): 517-530. Disponible en: http://scielo.isciii.es/scielo.php?script=sci_arttext\&pid=S1135$57272002000500013 \& \operatorname{lng}=\mathrm{es}$

Giddens, Anthony. (1991) Sociología. Alianza Editorial S.A. (Eds.), 515-524, Madrid 
Cámara de diputados del H congreso de la Unión. Ley Federal del Trabajo. 2015. Consultado el 23 de octubre de 2018 (Online). Disponible en: https://www.gob.mx/cms/uploads/attachment/file/156203/1044_Ley_Federal_de 1_Trabajo.pdf

Rodríguez, B. C., (2009) Exposición a peligros ocupacionales de los profesionales de enfermería de urgencias, unidad de cuidados intensivos y salas de cirugía del Hospital Universitario San Ignacio, enlace:http://www.javeriana.edu.co/biblos/tesis/enfermeria/20092/definitiva/tesi s21.pdf

Mesa de la torre, E, Et al. Valoración del riesgo psicosocial en las enfermeras de nefrología de los hospitales de Sevilla. Revista de la sociedad española de enfermería nefrológica. [Online] 2005;8(4): 1139-1375. Available from: http://scielo.isciii.es/scielo.php?script=sci_arttext\&pid=S1139-

13752005000400004 [Accessed 30 October 2018].

Bustillo, M, Et al. 1 Riesgo psicosocial en el personal de enfermería Servicio de urgencias en hospital universitario de cartagena. Revista Duazary. [Online] 2005; 12(1): 3240. Available from: https://dialnet.unirioja.es/descarga/articulo/5053070.pdf [Accessed 30 October 2018].

Ccooes. Ccooes. [Online]. Available

from: http://www.sanidad.ccoo.es/comunes/recursos/15617/doc142620_Guia_Basica_ de_riesgos_laborales_en_el_sector_sanitario.pdf [Accessed 23 April 2019].

Sousa, S.D.A.F. Riesgos psicosociales en el trabajo: estrés y estrategias de coping en enfermeros en oncología. Rev Latino-Am Enfermagem. [Online] 2013;21(6): 9. Available from: http://www.scielo.br/pdf/rlae/v21n6/es_0104-1169-rlae-02132365.pdf [Accessed 23 April 2019].

Arenas, S.A, Pinzón, A.A. Riesgo biológico en el personal de enfermería: una revisión práctica. Revista cuidarte. [Online] 2011;2(1): Available from: 
https://www.revistacuidarte.org/index.php/cuidarte/article/view/60/696 [Accesse d 30 October 2018].

Diaz, S.J., Ruiz, M. D.C., Riesgo biológico del profesional de enfermería en el servicio de emergencia del Hospital Daniel Alcides Carrión Callao, Perú. [Online] Rev enferm Herediana. 2017;10(1):54-62. Avaible From: http://www.upch.edu.pe/vrinve/dugic/revistas/index.php/RENH/article/downloa d/3132/3115 Acceso el 31 de Octubre de 2018

De Souza C dos S., Lima da Silva J.L., Antunes Cortez E., Schumacher K.P., Moreira R.C.S., De Almeida Nilson T.. Riesgos ergonómicos de lesión por esfuerzo repetitivo del personal de enfermería en el hospital. Enferm. glob. [Internet]. 2011 Jul [citado 2018 Nov 01]; 10( 23 ): 251-263. Disponible en: http://scielo.isciii.es/scielo.php?script=sci_arttext\&pid=S1695$61412011000300018 \& \operatorname{lng}=\mathrm{es}$. http://dx.doi.org/10.4321/S169561412011000300018 .

Ciercoles P. J. Riesgos biosanitarios del personal de enfermeria. (online) consultado el 23 de octubre 2017. Disponible en: https://www.codem.es/Adjuntos/CODEM/Documentos/Informaciones/Publico/7 e040f14-0bea-421f-b327-440fe67f3617/AD8496B2-1A69-4CE0-A056F532534F10DA/f9abfee0-1698-4d14-9c88-39ceac7fcf80/Riesgoslaborales.pdf

Elvio., F. T., 2015. El Empirismo En La Practica De Enfermería. Acta Medica Del Centro, 9(2), Pp. 63-70.

Abedl-rahman, F.E. The Effectiveness Of Marzano's Dimensions Of Learning Model In The Tenth Grade Students Acquisition Of Scientific Concepts In Science And The Development Of Critical Thinking Skills And Their Attitude Towards Science. British Journal of Education. [Online] 2017;5(1): 76-84. Available from: http://www.eajournals.org/wp-content/uploads/The-Effectiveness-of-MarzanosDimensions-of-Learning-Model-in-the-Tenth-Grade-StudentsAcquisition.pdf [Accessed 24 March 2019]. 
Mariale, M. CONNECTING BRAIN RESEARCH WITH DIMENSIONS OF LEARNING. Educacional Leadership. [Online] 2001;59(3):. Available from: http://beststudentdissertations.com/connecting-brain-research-with-dimensionsof-learning/ [Accessed 25 June 2019].

Thompson, M. An Evaluation of The Implementation of the Dimensions of Learning Program in an Australian Independent Boys School. International Education Journal . [Online] 1999;1(1): 45-60. Available from: https://openjournals.library.sydney.edu.au/index.php/IEJ/article/download/6747/ 7392\#page=49 [Accessed 25 June 2019].

Karim, S, Et al. Recostructing the Physics Teaching Didactic based on Marzano's Learning Dimension on Training the Scientific Literacies.Journal of Physics: Conference Series. [Online] 2017;812(1): 1-8. Available from: https://iopscience.iop.org/article/10.1088/1742-6596/812/1/012102/pdf [Accessed 25 June 2019].

Dujari, A.S. The Effect of Two Components of the Dimensions of Learning Model on the Science Achievement of Underprepared College Science Students. ERIC. [Online] 1994;ED379158( 055 775): 1-34. Available from: https://files.eric.ed.gov/fulltext/ED379158.pdf [Accessed 25 June 2019].

Franco C., SA, Serrano, LP, Vázquez G., JM, García P., LJ. RIESGO DE TRABAJO OCURRIDOS CON PERSONAL DE UN HOSPITAL EN EL ESTADO DE JALISCO 2009. e-Gnosis [Internet]. 2012;10:1-26. Recuperado de: http://www.redalyc.org/articulo.oa?id=73026905004

Bustillo, M, Et al. 1 Riesgo psicosocial en el personal de enfermería Servicio de urgencias en hospital universitario de cartagena. Revista Duazary .[Online] 2005; 12(1): 3240. Available from: https://dialnet.unirioja.es/descarga/articulo/5053070.pdf [Accessed 30 October 2018].

McAnally Salas, L. Diseño educativo basado en las dimensiones del aprendizaje. Apertura [Internet]. 2005;5(1):31-43. Recuperado de: http://www.redalyc.org/articulo.oa?id=68850104 
Pecina leyva, R.M. Impacto de la educación basada en competencias en el aprendizaje de alumnos de octavo semestre de licenciatura en enfermería en una universidad pública. Revista Iberoamericana para la Investigación y el Desarrollo Educativo. 2013; Desconocido (10): 1-20.

Masoudi Alavi, Negin. "Occupational hazards in nursing." Nursing and midwifery studies (internet) vol. 3,3 (2014): e22357.(Acceso 30 de Octubre de 2018). Disponible en: https://www.ncbi.nlm.nih.gov/pmc/articles/PMC4332998/

Garduño SA., Mendez SV., Salgado GJ., Autocuidado del Profesional de Enfermeria en un Hospital Público. Articulo Original. (internet). 2012:5:2:19-27. Recuperado de:

http://web.uaemex.mx/revistahorizontes/docs/revistas/Vol5/2_AUTOCUIDADO .$p d f$

Elvio., F. T., 2015. El Empirismo En La Practica De Enfermeria. Acta Medica Del Centro , 9(2), Pp. 63-70.

Collado, E. F., 2005. Universidad Europea Madrid. [En Línea] Available At: Http://Abacus.Universidadeuropea.Es/Handle/11268/1435 [Último Acceso: 2 Septiembre 2018].

Consejo internacional de enfermeras. The global nursing shortage: Priority areas for intervention [Internet]. Ginebra: Consejo internacional de enfermeras; 2004 [estado el 20 de abril de 2017]. Disponible: http://www.icn.ch/images/stories/documents/publications/GNRI/The_Global_N ursing_ShortagePriority_Areas_for_Intervention.pdf

Franco C., SA, Serrano, LP, Vázquez G., JM, García P., LJ. RIESGO DE TRABAJO OCURRIDOS CON PERSONAL DE UN HOSPITAL EN EL ESTADO DE JALISCO 2009. e-Gnosis [Internet]. 2012;10:1-26. Recuperado de: http://www.redalyc.org/articulo.oa?id=73026905004

Valente, G. Y Viana, L. (2007). El Pensamiento Crítico-Reflexivo En La Enseñanza De La Investigación En Enfermería: ¡Un Desafío Para El Profesor!. Enfermería 
Global, [En Linea $]$ 6(1), Pp.1-7. Disponible En: Http://Www.Redalyc.Org/Articulo.Oa?Id=365834734012 